\title{
Towards Learning Social Cultures through Virtual Reality Game
}

\author{
Karuna Yampray $^{+}$and Waraporn Jirapanthong \\ College of Creative Design and Entertainment Technology, Dhurakij Pundit University, Thailand
}

\begin{abstract}
Bringing games to support in education is not nonsense anymore. Naturally, games are an obvious terrain in which to set a player's mind free and let him wander around. In this paper, our game is created and used to support a player to learn Thai culters. The player is allowed to interact with objects and game-characters he encounters in order to learn social rules and procedures in each location. In this paper, we presents a game, entitled "Know more Thai", which is a Virtual Reality game for players to learn about Thai Etiquette and Customs. More specifically, it is aimed to support foreigners who visit Thailand and go to attraction places. Basically, the visiting the attraction places are ruled by Thai history and believes. In particular, the game allocated the location of Wat Pra Kaew and Royal Palace which are the very important place in Thailand. The rules and instructions for visiting the place are disciplined in the game. There are officers patrolling to monitor the situations.
\end{abstract}

Keywords: virtual reality, augmented reality, gamification, active learning, Unity3D

\section{Introduction}

Virtual reality (VR) is a popular information technology that has been evolving for providing an indirect experience by creating a virtual space. VR technology enables us to create a virtual space that interacts with the human sensory systems and overcomes spatial and physical constraints of the real world (Electronics and Telecommunications Research Institute (ETRI), 2001, 2010). In fact, the concept of virtual reality has been developed for years and it is applied in various domains. For example, the VR experiences provide for a controlled environment in which patients can face their fears and even practice coping strategies, as well as breaking patterns of avoidance. VR experiences can provide another means of surgery practice without any risk to real patients [1]. Moreover, there are two ways of using VR in the classroom [2], [3]: i) it is applied with a traditional computer desktop that allows a student to explore a virtual environment using a computer, keyboard, mouse, and other devices; ii) it requires a student to wear a head mounted display (HMD) and data glove for interaction within a virtual environment. The virtual environment takes many forms of large screens as CAVE virtual reality [4]. It is an ideal way of engaging the students with a particular subject in a manner they are comfortable with.

In this paper, we propose "Know more Thai", a Virtual Reality game for foreigners to learn about Thai Etiquette and Customs. We planned to evaluate the result of learning through the game by a case study. In particular, the case study is being developed in order to find out how the VR game can enable the foreigners understanding and acknowledging the knowledge of Thai Etiquette. The information about procedures and rules are presented in a virtual environment. In this paper, we present the design of the VR game "Know more Thai" that provides a channel to learn more about Thai Etiquette and custom. The design of this game has been divided into two parts: i) Visiting the Grand Palace, and ii) the temple of the Emerald Buddha Etiquette. As believed, VR games become the absolutely most immersive learning technology that gives learners a powerful sense of presence in the virtual environment. The technology has been rapidly adopted and heavily invested in by a wide variety of training in both hard and soft skills. In this work, we combine

+ Corresponding author. Tel.: +66809055775.

E-mail address: karuna.yam@dpu.ac.th. 
the aspects of learning the knowledge and of entertaining through the virtual reality environment. To evaluate the learning through the game, we create a survey consisting with 60 participants who are foreigners and have no knowledge regarding the Thai customs.

\section{Background and Related Work}

\subsection{Virtual Reality}

Currently, many vendors have provides the baseline of technologies to support VR applications. For example, Google provides developers with two VR platforms: i) Cardboard which is accessible mobile VR platform, and ii) Daydream which is a new low-latency, immersive, and interactive mobile VR. Google also provides software development kit for various APIs such as Unity, Android, and iOS. Moreover, Oculus [5] provides the platform called Oculus. Oculus also provides supporting kits for Coordinated App Launch (CAL).

A lot of work have been evolved to apply VR technology for supporting education and learning. For example, Donncha and others [6] at Royal College of Surgeons in Ireland (RCSI) developed the interactive VR medical training simulator which allows users simulate emergency room management of a patient following a road traffic accident. It is called RCSI VR Medical Training Sim application that puts medical professionals and trainees as the Emergency Department trauma team leader. They must access the patient, make decisions in real time and perform life-saving operative procedures as a surgeon in a real emergency room. Some of RCSI VR systems include a hybrid laparoscopic trainer which takes an ordinary box trainer and transforms it into an experience in an operating theatre. It can simulate the stressful and immersive environment for virtual operation experienced by young doctors. ATi Studios [7] developed an application for learning languages with Mondly VR. The application is available on iPhone, iPad, Android and PC. It provides 28 languages and allows a learner experienced on learning languages and getting feedback on his pronunciation and suggestions.

\subsection{Gamification}

Gamification commonly employs game design elements which are used in non-game contexts to improve user engagement, organizational productivity, flow, learning, crowdsourcing, employee recruitment and evaluation, ease of use, usefulness of systems, physical exercise, traffic violations, voter apathy, and more [12].

Many work have applied gamification to support education and learning areas. For example, Microsoft released the game Ribbon Hero $2^{1}$ as an add-on in their software package. It is used to train people to use it effectively. SAP has used games to educate their employees on sustainability [8]. Also, the Khan Academy applies gamification techniques in online education [9]. The use of gamification also appears in various situations. Gbanga launched the educational location-based game Gbanga Zooh for Zurich Zoo that asked participants to actively save endangered animals and physically bring them back to a zoo. Players can learn virtual habitats across the Canton of Zurich to attract and collect endangered species of animals [10]. There is some indication that gamification can be particularly motivational for learners with dyslexia in educational situations.

\subsection{Unity3D}

Unity is one of the most famous virtual reality cross-platform tool for game development software. Unity $3 \mathrm{~d}$ Game engine is a sharp weapon to develop 3D game, real time 3D animation and other interactive projects. The most impressive function is the powerful trans- platform ability. It is an engine compatible with all stream platforms such as Windows and MAC OS systems. It can also work with PC, iPhone, android, MAC, Wii, Windows phone, PlayStation, Xbox, etc. Unity supports Microsoft Visual Studio and Mono Development. User can create a script programming on these two development tools [11]. An application which is built by Unity application is enabled with complete 3D environment, laying out levels, creating menus, doing animation, writing scripts, and organizing projects. The user interface is well organized and the panels can be fully customized by dragging and dropping.

\footnotetext{
${ }^{1}$ www.ribbonhero.com
} 
Moreover, assets are any resource in a game. Those can be 3D models, materials, textures, audio, scripts, and fonts. An object can be appeared in various shape such as cube and sphere. The assets can be created externally using 3D modelling applications and painting tools and then imported into Unity. Traditionally, 3D game engines have usually been selective things and are very particular about what files a developer gives them. The developer is forced to carefully convert all non-Unity files. Currently, it accepts 3D file formats including Maya, 3D Studio Max, Blender and FilmBox with all the rigging, materials and textures intact. Unity also supports all common image file formats, including PNG, JPEG, TIFF and even layered PSD files directly from Photoshop. For audio files, Unity supports WAV and AIF, ideal for sound effects, and MP3 and OGG for music.

\section{The Prototyping Game for Learning Thai Etiquette and Culture for Foreigners}

The main objectives of the game are: i) to provide a learning channel about Thai Etiquette and Culture for foreigners, and ii) to entertain a player as a VR game. The design of game focuses on the learning of history and background of main attractions in Thailand, and Thai cultures. In particular, the game allocated the location of Wat Pra Kaew and Royal Palace which are the very important place in Thailand. The rules and instructions for visiting the place are disciplined in the game. There are officers patrolling to monitor the situations. As shown in Figure 1, the design storyboard of game.
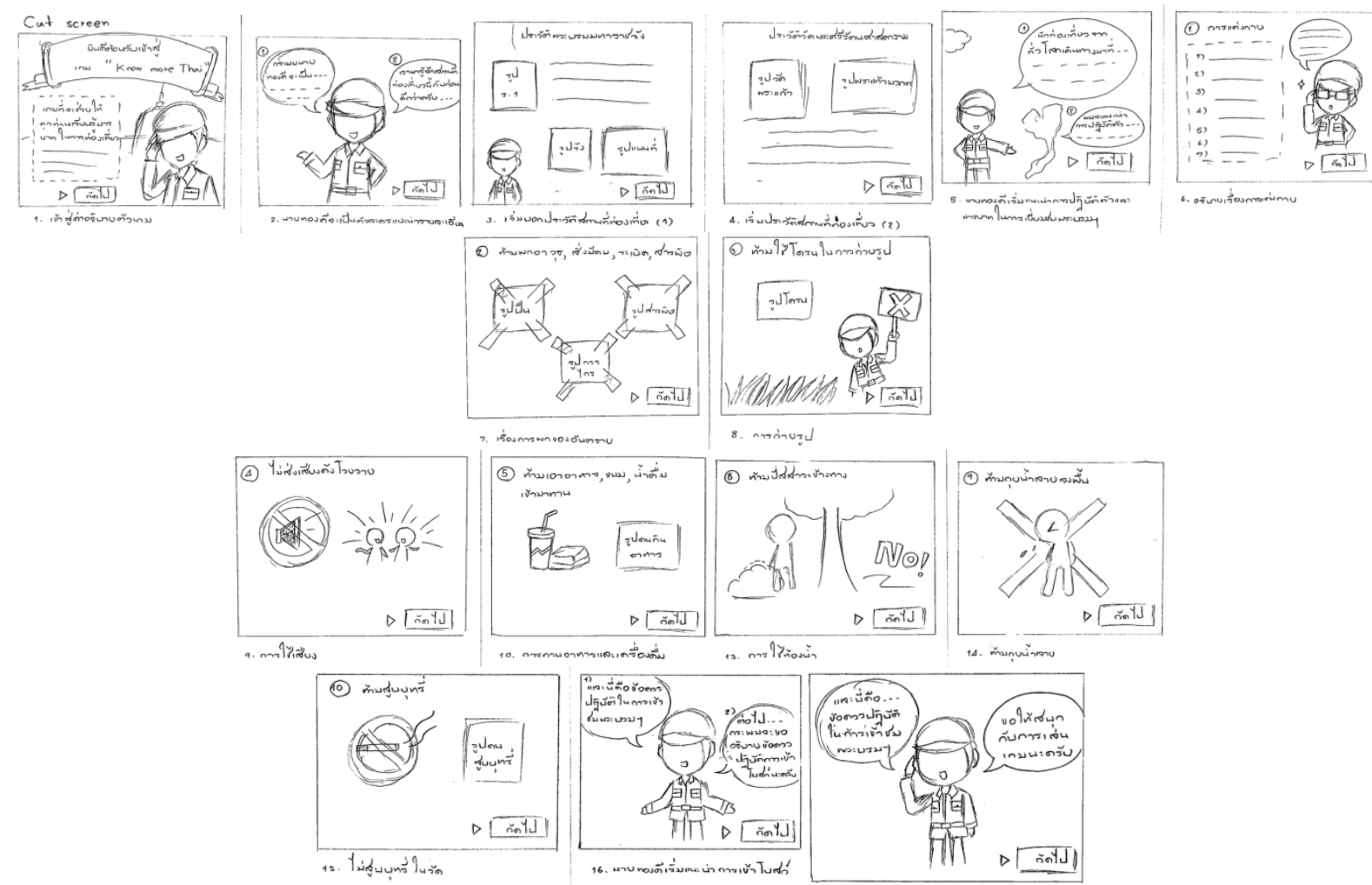

Fig. 1: A part of design storyboard.
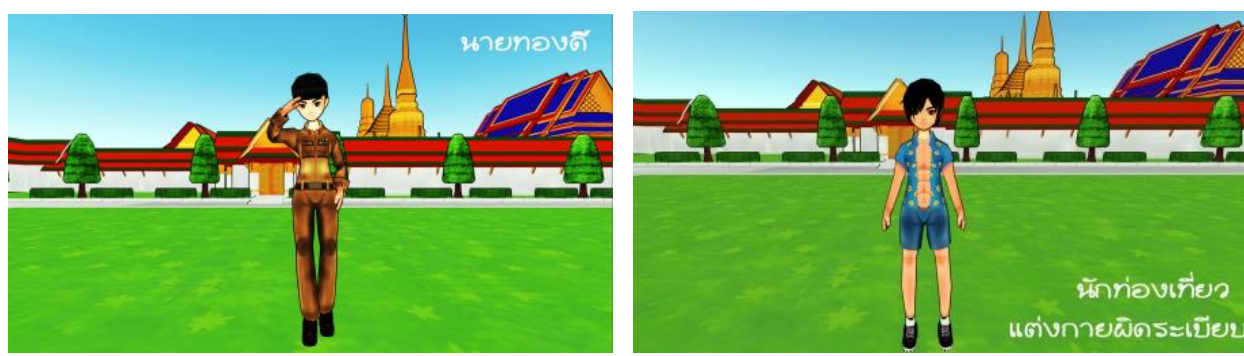

Fig. 2: Examples of 3D characters with 1:2 proportion. 
In each scenario the constraints and rules are embedded to allow a player to learn Thai Etiquette and Culture through the game. According to the storyboard, the design is included the series of scenarios in the story and playing style in the game.

In the game, a player can play as a character and control it with a wireless remote control. The player can take actions in the game and make a move within the third party's perspective. The environment in the game is up to 60 degrees of camera view. Some examples of game scenarios are shown in Figure 2.

We apply 3Ds Max modelling for creating a game design model and design the characters by the ratio of 1:2 or SD (Super Deformation). This enables the character form with the unbalanced proportion of character body. Specifically, the size of character's head is outstandingly larger than its body [9]. Moreover, the physical reality of each scenario is embedded in term of 3D modelling. To do this, we have done field study in main locations such as Wat Pra Kaew and Royal Grand Palace.

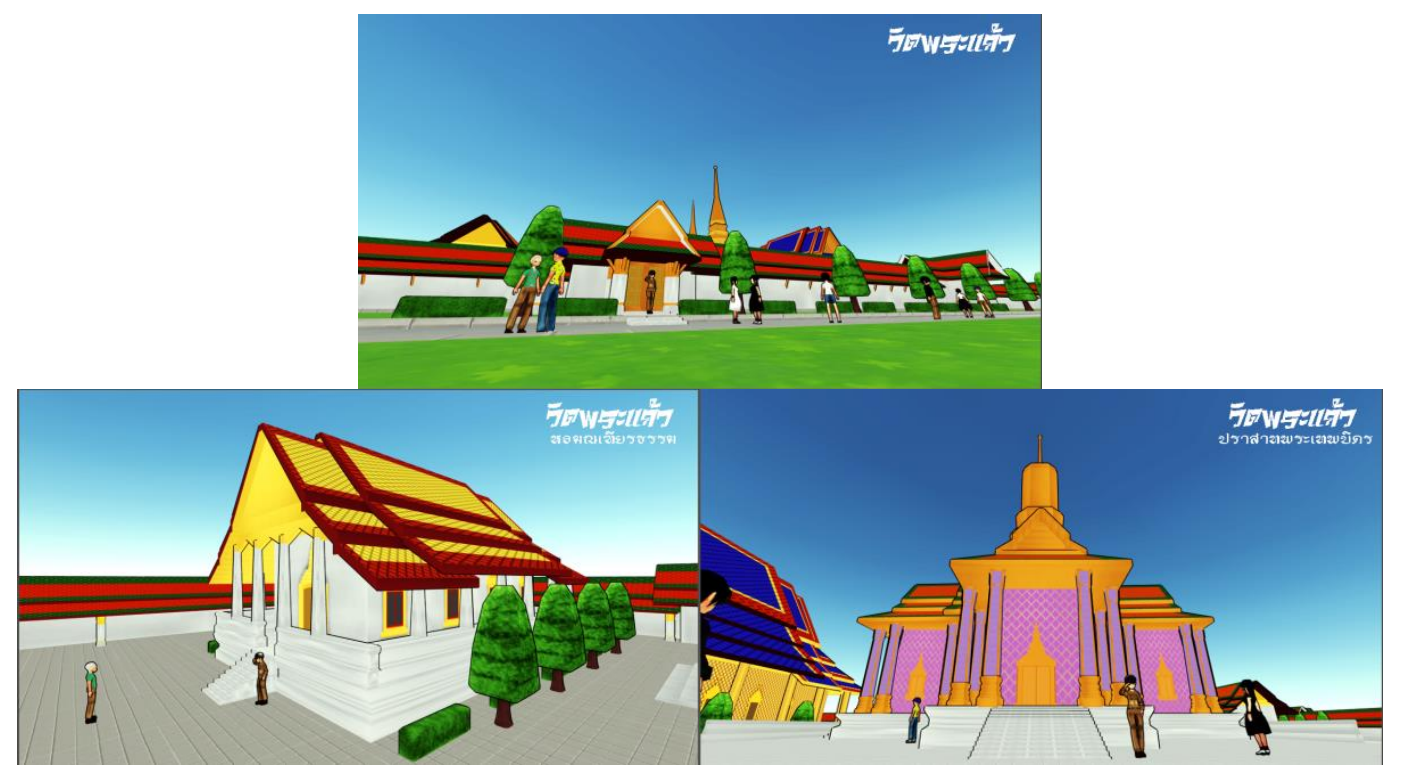

Fig. 3: Examples of VR design for game environment (Wat Pra Kaew).

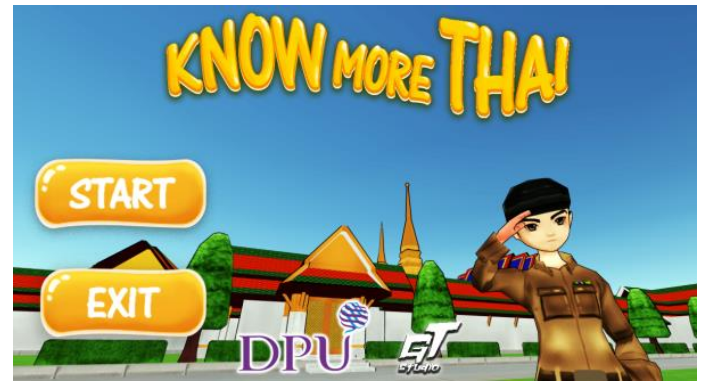

Fig. 4: The home scene of the game.

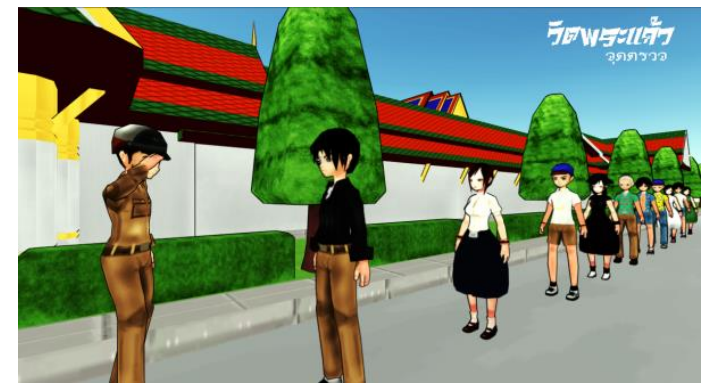

Fig. 5: The game scene "Know More Thais".

The game consists of 3 parts: i) a part of characters that are presented in term of 3DMax cartoons, ii) a part of screens, and iii) a part of game architecture in term of 3D model. The model is developed based on real ratio by Unity 3D and integrated with sound by Adobe Audition programs. The game program is developed based on Unity 3D program and MS Window console platform. It is also available on iOS and Android platforms.

As shown in Figure 2, the cartoon character named "Thong-Dee" is a main character in the game and responsible for explaining Thai Etiquette and Custom when people visit an attraction place in Thailand. In the game, we have two main locations, Wat Pra Kaew and Royal Grand Palace. Figure 3 show the architectural model of attraction points in Royal Grand Palace which appears as main cut-scene in the game. Figures 4 and 5 are the main screen of the game and the start of the game, respectively. The main objectives 
of this game are to i) evaluate the Virtual Reality game about Thai etiquette and customs for the travellers, and ii) enhance the knowledge about Thai etiquette and customs for the travellers.

\section{Conclusion and Discussion}

A number of possible directions for further investigations have been identified. We provide in this section future work of the research, what needs to be done to improve the approach and to increase the benefits of the approach. We intend to extend the game to cover all scenarios of attraction places in Thailand. The scenarios of game would provide more lessons to a player. It is therefore believed that the approach could benefit by providing more lessons and fully support for the education. In addition, sophisticated techniques for visualization could support the use of learning materials more efficiently.

\section{References}

[1] Carson, E. (2015). Virtual reality spotlight: What can VR bring to the enterprise?. Retrieved February 6, 2017, from Tech Pro Research Web site: http://www.techproresearch.com/downloads/virtual-reality-spotlight-what-canvr-bring-to-the-enterprise/.

[2] Reede, E. and Bailiff, L. (2016). When Virtual Reality Meets Education. Retrieved January 22, 2017, from TC Crunch Network Web site: https://techcrunch.com/2016/01/23/when-virtual-reality-meets-education/.

[3] Anonymous. (2016). Virtual Reality. Retrieved January 22, 2017, from Wikipedia Web site: https://en.wikipedia.org/wiki/Virtual_reality.

[4] Virtual Reality Society. (2017). CAVE Fully Immersive Virtual Reality. Retrieved February 6, 2017, from Virtual Reality Society Web site: https://www.vrs.org.uk/.

[5] Rubin, J. (2017). The State of the Game: VR in 2017. Retrieved March 9, 2017, from Oculus Web site: https://www.oculus.com/blog/the-state-of-the-game-vr-in-2017/.

[6] Shen, L. et. al. (2012). SD Models: Super-Deformed Character Models. Pacific Graphics. Vol. 31, No. 7.

[7] Fan, Z. and Wenfeng, H. (2013). The game development foundamental of Unity 3D. Zhejiang Gongshang University Press.

[8] Corbett, S. (2010). Learning by Playing: Video Games in the Classroom. Retrieved November 13, 2016, from The New York Times Web site: http://www.nytimes.com/2010/09/19/magazine/19video-t.html.

[9] Herger, M. (2011). Sustainability examples. Retrieved November 13, 2016, from Enterprise Gamification Web site: https://Enterprise-Gamification.com.

[10] Sinha, S. (2012). Motivating Students and the Gamification of Learning. Retrieved November 13, 2016, from Huffington Post Web site: http://www.huffingtonpost.com/shantanu-sinha/motivating-students-andt_b_1275441.html.

[11] Deterding, S. et. al. (2011). From Game Design Elements to Gamefulness: Defining "Gamification”. In Proc. of the 15th International Academic MindTrek Conference: Envisioning Future Media Environments, Tampere, Finland, September 28-20, 2011. pp. 9-15. 